\title{
Acute haemorrhagic leucoencephalitis localised to the brainstem and cerebellum: a report of two cases
}

\author{
JEAN MICHAUD, TODD L HELLE \\ From the Department of Pathology (Neuropathology), Stanford University Medical Center, Stanford, \\ California, USA
}

SUMmary Two cases of acute haemorrhagic leucoencephalitis localised to the brainstem and cerebellum are reported. One followed the insertion of a ventriculoatrial shunt and the other an upper respiratory tract infection. The rare previously reported cases of this condition involving mainly the posterior fossa structures are reviewed.

Hurst $^{1}$ was the first to define acute haemorrhagic leucoencephalitis as a clinicopathological entity. He distinguished it from other brain purpuras and haemorrhagic encephalitides, and regarded it as an acute demyelinating condition. Occurring mainly in adults, it usually follows an upper respiratory tract infection but may occur without any prodromal illness. The onset is abrupt, with pyrexia, leucocytosis and a variety of neurological symptoms including severe headaches, seizures and paralysis. It usually follows a fulminant course, with coma occurring in a few hours, and death ensuing several hours to a few days later. ${ }^{2} 3$ Survivals, ${ }^{245}$ or deaths after a more chronic course ${ }^{4} 6$ are rare.

The pathological changes are usually found in the white matter of the cerebral hemispheres, often asymmetrically. They may extend into the brainstem and cerebellum, but they only rarely involve these structures exclusively. In a period of five months we encountered two cases implicating almost exclusively the brainstem and cerebellum. These two cases form the basis of this report.

\section{Case reports}

CASE 1

Clinical history This 82-year-old white female was admitted because of hydrocephalus. Five years before admission, she developed a progressive gait disturbance with signs of Parkinsonism. Two years later computed tomography (CT scan) showed changes strongly suggestive of hydrocephalus. She responded well to low

Address for reprint requests: Dr J Michaud, Département de Pathologie, Faculté de Médecine, Université de Montréal, CP, 6128, Succursale "A", Montréal, PQ, Canada H3C 3J7.

Accepted 21 July 1981 doses of carbidopa and levodopa. Eleven months before admission she noticed increasing difficulty swimming and diving. Another CT scan showed further dilatation of the ventricles and a radioisotope cerebrospinal fluid (CSF) study showed reflux in the ventricles, with delayed absorption over the convexities. Six months before admission she showed minimal signs of senility, gait apraxia and intermittent incontinence. She also had a bizarre voice disturbance with oral facial dyskinesias, probably resulting from anti-parkinsonism medication. She denied any history of headaches or visual disturbances. Progressive deterioration in her neurological status prompted her final admission. Her past medical history included only mild arteriosclerotic heart disease. No history of allergy was elicited. At the time of her admission her medication consisted of carbidopa, levodopa, digoxin, clorazepate, amantadine, and Donnatal (Robins).

General examination revealed a temperature of $36.7^{\circ} \mathrm{C}$, blood pressure of $140 / 60 \mathrm{mmHg}$, and a pulse rate of $100 / \mathrm{min}$. There was a soft systolic ejection murmur over the aortic area. The neck was supple. Neurological examination showed a normocephalic, mildly confused woman oriented to person, place, and time, with mild dysarthria and mild dyskinesias, no papilledema, and definite rigidity associated with mild cogwheeling of the upper limbs. She could not sit, stand, or walk without assistance. Tendon reflexes were decreased in the lower limbs. Plantar reflexes were flexor. A mild hyperaesthesia was noted on the left side of the body excluding the face. Laboratory tests, electrocardiogram, and chest radiograph were normal. CT scan showed changes similar to those found eleven months before admission.

A right ventriculoatrial shunt was inserted and 4 $\mathrm{mg}$ of gentamicin were injected into the ventricles through the catheter. The first day after the operation was unremarkable. On the second day she complained of a sudden severe headache. This was followed by a sharp decrease in mentation, breathing difficulties, stridor, and tachycardia necessitating intubation. She had a grand 
mal seizure and her left pupil dilated. Hyperventilation was instituted, and phenytoin and mannitol were given intravenously. Her left pupil then became normal. An emergency CT scan showed that the lateral ventricles were slightly smaller than prior to surgery. The catheter was in good position and there were no haemorrhages. She had another seizure and her temperature rose to $38.5^{\circ} \mathrm{C}$. Her WBC was $39.6 \times 10^{\%} / 1$ with $69 \%$ polymorphs and $18 \%$ bands. The following day her temperature remained elevated, up to $39 \cdot 5^{\circ} \mathrm{C}$. She could open her eyes but did not respond to verbal commands. She could move her legs, the left more than the right, but no movements of the arms could be elicited. Pupils were equal and reactive to light. CSF obtained from the shunt revealed a RBC of $6.5 \times 10^{12} / 1$, WBC of $108 \times 10^{6} / 1$ with $90 \%$ polymorphs and $10 \%$ mononuclear cells, glucose of $5.2 \mathrm{mmol} / 1$ and protein of $1.62 \mathrm{~g} / \mathrm{l}$. Antibiotics were started. CSF cultures and cultures from the septum, urine, and blood were negative. A chest radiograph revealed a right middle lobe infiltrate consistent with atelectasis or bronchopneumonia. Three days after operation she was successfully extubated and her chest radiograph cleared. During this time her temperature varied from $37 \cdot 5^{\circ} \mathrm{C}$ to $39 \cdot 0^{\circ} \mathrm{C}$. She began to obey simple commands. On the eighth day after operation her temperature rose sharply to $39.7^{\circ} \mathrm{C}$. She had another seizure, and a chest radiograph again showed a right middle lobe infiltrate. Over the next hours she progressed rapidly to coma and died.

\section{Necropsy findings}

General pathology: Both lungs showed acute congestion, extensive acute bronchitis with mucus plugging, and patchy bronchopneumonia of the lower lobes. Focal scarring and calcified granulomas were found in both pulmonary apices. The right cardiac ventricle was dilated and there was an acute congestion of the abdominal viscera. Two broad and shallow duodenal ulcers were found but no significant amount of blood was seen in the intestinal lumen. Myeloid hyperplasia was noted in the bone marrow.

Neuropathology: the brain weighed $1200 \mathrm{~g}$. The ventriculoatrial shunt, which was patent, extended from the right posterior parietal convexity and was surrounded by a small amount of meningeal haemorrhage. The leptomeninges of both cerebral hemispheres were otherwise clear and the hemispheres were symmetrical. The posterior fossa structures were swollen and soft. The cerebellar tonsils were herniated and necrotic, more so on theleft side. The meninges were congested and a yellow mottling was seen over the brainstem. The vessels of the circle of Willis showed mild patchy non-occlusive atherosclerosis. Multiple coronal sections of the cerebrum revealed a moderate enlargement of the lateral and third ventricles. There was no cortical atrophy. The white matter was unremarkable except for the shunt track. The tip of the catheter was located in the body of the lateral ventricle, which showed focal haemorrhagic discoloration of its wall. Multiple transverse sections of the brainstem and cerebellum showed a diffuse softness with numerous petechiae and confluent haemorrhages involving the midbrain, pons, medulla and the white matter of both cerebellar hemispheres, more so on the left side (fig 1). In the inferior vermis, a small haematoma measuring $1.5 \mathrm{~cm}$ in diameter was found. The substantia nigra was well pigmented. Multiple transverse sections of the spinal cord were unremarkable.

Microscopic examination: representative sections were stained with one or more of the following stains: haematoxylin and eosin (HE), Mallory's phosphotungstic acid haematoxylin (PTAH), Gram, periodic acid Schiff (PAS), Gomori's methenamine silver (GMS), luxol fast blue-cresyl violet (LFB-CV), phloxine-fast greengallocyanin (PFG) ${ }^{7}$ and Bielschowsky's silver impregnation for axons. ${ }^{8}$ Numerous ball and ring haemorrhages were seen in the brainstem and in the white matter of the cerebellum (fig 2). They were centred by small veins and occasional arteries which frequently showed complete fibrinoid necrosis of their walls. There were also perivascular inflammatory exudates composed mostly of polymorphonuclear leukocytes with rare mononuclear

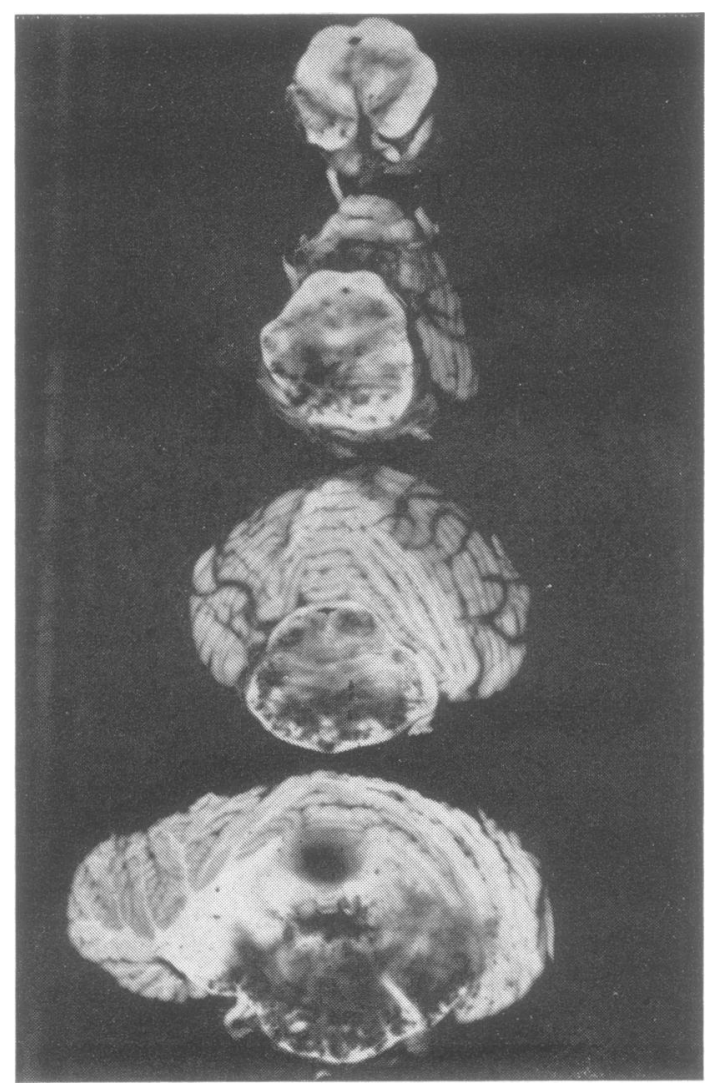

Fig 1 Case 1. Transverse sections of the midbrain, pons and cerebellum (left side on the right) showing numerous petechiae and confluent haemorrhages. 


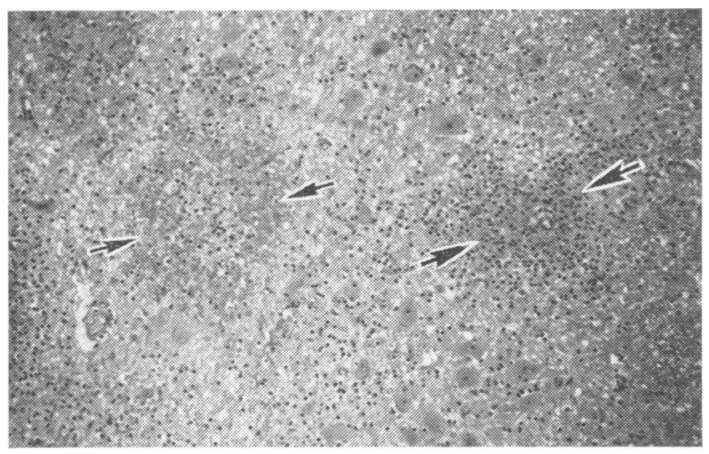

Fig 2 Case 1. Photomicrograph of the midbrain tegmentum showing ring haemorrhage (small arrows), perivascular inflammation (large arrows) and oedema. $H E \times 120$.

cells (fig 3a). In the white matter of the cerebellum some foci were less haemorrhagic, and the exudates contained more mononuclear cells and less polymorphonuclear leucocytes. The PTAH stain confirmed the fibrinoid necrosis of several small veins and arteries and showed also fibrin exudates in the perivascular space and neural parenchyma (fig $3 \mathrm{~b}$ ). Small areas of myelin loss with

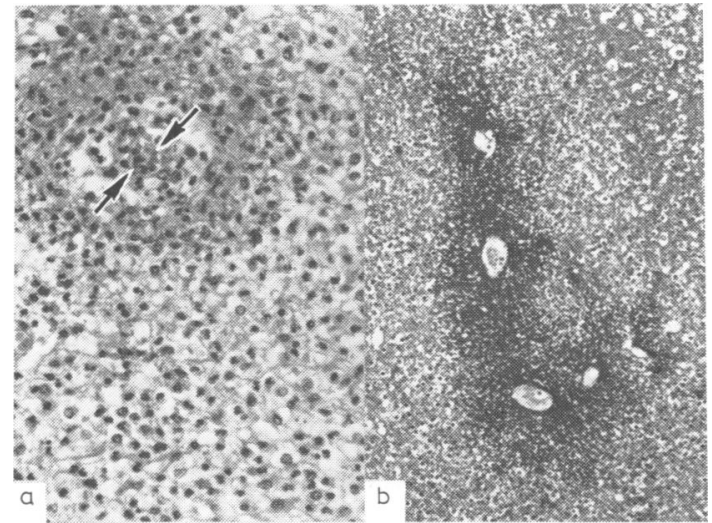

Fig 3 Case 1. (A) Photomicrograph of the pontine tegmentum showing fibrinoid exudate and acute inflammation around a blood vessel. The lumen only is clearly visible (arrows). $H E \times 300$. (B) Photomicrograph of the basis pontis showing fibrinoid necrosis of several blood vessel walls and perivascular fibrinous exudates. $P T A H \times 120$.

minimal axonal damage were present around several small veins, not always associated with fibrinoid necrosis or haemorrhages (fig 4). There were also several foci of necrosis in the parenchyma, and more diffuse oedema. The meninges contained a patchy mild to moderate acute inflammatory exudate. No organisms were demonstrated. In the cerebellum, the fibrinoid necrosis of small veins and arteries, and the perivascular inflam- mation, haemorrhages and myelin loss were mostly localised to the white matter, but rare foci of perivascular inflammation involved the dentate nuclei. In the midbrain, the cerebral peduncles, substantia nigra and tegmentum were severely involved, while the tectum and red nuclei showed only rare foci of perivascular inflammation. The vascular fibrinoid necrosis, acute inflammation and haemorrhages were very severe in the pontine tegmentum, and in the grey nuclei and fibre tracts of the basis pontis. In the medulla, the rostral tegmentum was more severely involved. Occasional foci of perivascular inflammation were seen in both pyramids and in the area postrema.

Outside the posterior fossa, no similar lesions were found, but a few focal acute inflammatory exudates were seen in the meninges at the base of the brain and along the spinal cord. The latter was otherwise normal. The hippocampi showed numerous neurofibrillary tangles, a moderate number of senile plaques and occasional neurons with granulovacuolar degeneration. Occasional senile plaques were seen in the frontal cortex. There were a few small foci of gliosis in the right striatum. Along the shunt tract there were necrosis, focal haemorrhages and chronic perivascular inflammation.

\section{CASE 2}

Clinical history This 39-year-old white male was admitted drowsy one week after the onset of fever, nausea, vomiting, headache and general malaise. His past medical history included moderate alcohol and marijuana use, and a serious motor vehicle accident 12 years before admission. On general examination his temperature was $39 \cdot 2^{\circ} \mathrm{C}$, blood pressure was $130 / 60$ $\mathrm{mmHg}$, pulse was $120 / \mathrm{min}$, and respiratory rate was $20 / \mathrm{min}$. There was a II/VI systolic cardiac murmur heard best over the apex. There was no nuchal rigidity. Neurologically he was somnolent, had slurred speech, and was oriented only to person. His pupils were small and reactive to light, and there was no papilloedema. $\mathrm{He}$ had generalised hyperreflexia and bilateral Babinski

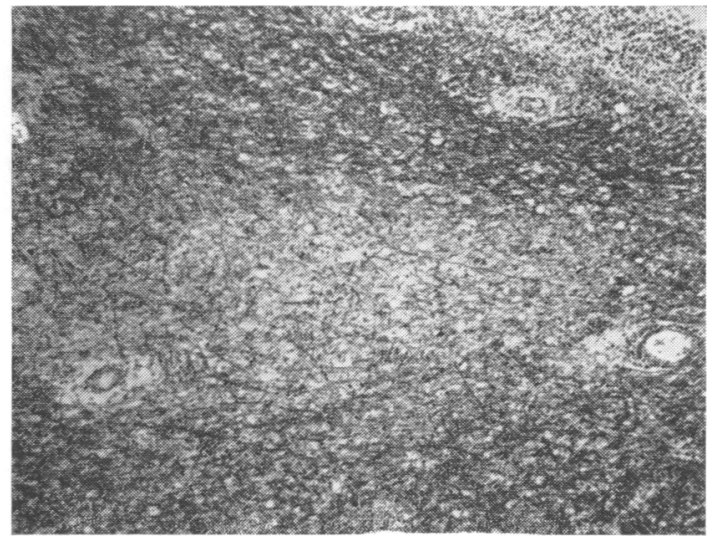

Fig 4 Case 1. Photomicrograph of the left cerebellar white matter showing a focus of demyelination between two small blood vessels surrounded by mild chronic inflammation. $L F B-C V \times 75$. 
reflexes. Laboratory tests revealed a WBC of $16.6 \times 10^{9} / 1$ with $91 \%$ polymorphs and $4 \%$ bands. His haematocrit, platelet count, electrolyte, serum glucose, urinalysis, and chest radiograph were normal. The blood alcohol level was zero. A lumbar puncture was performed and revealed CSF with a protein of $1.1 \mathrm{~g} / \mathrm{l}$, glucose of 5.0 $\mathrm{mmol} / \mathrm{l}, \mathrm{RBC}$ of $0.5 \times 10^{12} / \mathrm{l}$, and a WBC of $5 \times 10^{6} / 1$, all polymorphs. India ink, Gram stain, and subsequent cultures were all negative. Intravenous chloramphenicol and penicillin were started. Approximately one hour after lumbar puncture the patient had respiratory arrest. After resuscitation, he was hypotensive, apnoeic, unresponsive, and flaccid with fixed midpositioned pupils. Hyperventilation was started, and dexamethasone, mannitol, and furosemide were given intravenously. CT scan revealed severe hydrocephalus involving the lateral and third ventricles. The posterior fossa was full, and there was a low density, nonenhancing lesion of the upper brainstem and deep cerebellar tissues distorting and shifting the fourth ventricle to the right. The patient underwent an emergency ventriculoperitoneal shunting. Postoperatively there was no clinical improvement. No brainstem reflexes could be elicited and the EEG was isoelectric. He died approximately 18 hours after being seen in the emergency department.

\section{Necropsy findings}

General pathology: All viscera were congested. There was pulmonary oedema and early bronchopneumonia. Small congenital fenestrations were present in the aortic and pulmonary valve leaflets. Mild generalised arteriosclerosis was present.

Neuropathology: the brain weighed $1650 \mathrm{~g}$. The ventriculoperitoneal shunt catheter extended from the right parietal convexity. The cerebral hemispheres were symmetrical and mildly swollen, with scattered small subarachnoid haemorrhages. The posterior fossa structures were markedly swollen, with upward transtentorial and downward tonsilar herniation. The interpeduncular cistern was obliterated. The surface of the cerebellar hemispheres was soft and friable, and appeared ragged. The blood vessels of the circle of Willis and the spinal cord were unremarkable.

Multiple coronal sections of the cerebral hemispheres revealed diffuse mild oedema and disclosed the shunt tract in the parietal lobe. There was a small haemorrhage in the head of the right caudate nucleus. A small amount of blood was present in the lateral and third ventricles. Transverse sections of the brainstem and cerebellum showed brown mottled haemorrhagic discoloration of the pons and medulla and, to a lesser extent, of the middle cerebellar peduncles and the vermis (fig 5 ). There was a well defined haemorrhage in the left midbrain tegmentum. The superior vermian cistern was obliterated by blood. The cerebellar cortex was soft and very fragmented. Multiple transverse sections of the spinal cord were unremarkable.

Microscopic examination: representative sections were stained with one or more of the following stains: HE, PTAH. Loyez, LFB-CV, Gram, PAS, GMS and PFG. Numerous small veins and arteries with segmental or

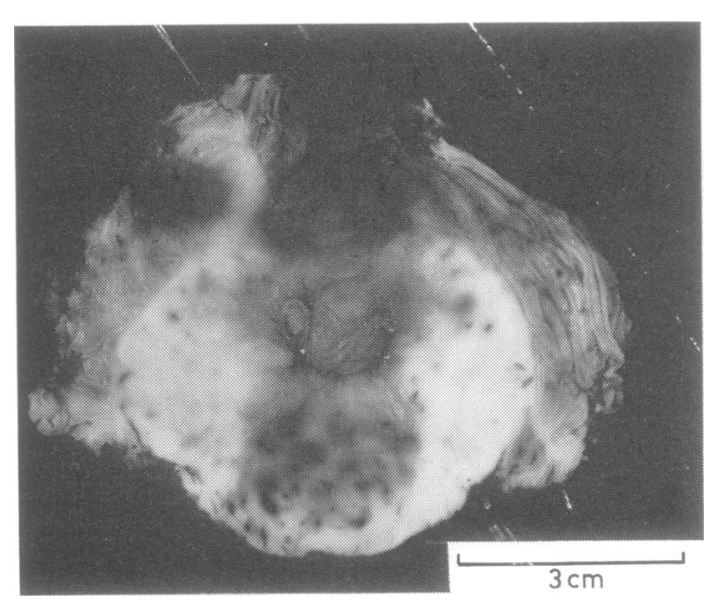

Fig 5 Case 2. Transverse section of the pons and cerebellum showing petechiae, confluent haemorrhages and oedema, with obliteration of the fourth ventricle.

complete fibrinoid necrosis of their walls were seen in the brainstem, middle cerebellar peduncles and cerebellar vermis. Several small veins and arteries were surrounded by ball or ring haemorrhages which were confluent in places. There were also perivascular acute inflammatory exudates composed nearly exclusively of polymorphonuclear leucocytes. Around some blood vessels with fibrinoid necrosis, the inflammation was scanty and the haemorrhages were absent, suggesting very early necrotic changes. The PTAH stain confirmed the fibrinoid necrosis of the walls of several small veins and arteries and revealed fibrin exudates in the surrounding perivascular spaces and neural parenchyma. Myelin stains showed discrete foci of myelin loss without axonal damage around several small veins, occasionally extending beyond the site of inflammation or haemorrhages (fig 6). There was also diffuse oedema and several foci of necrosis.

The vascular fibrinoid necrosis, and perivascular acute inflammation, haemorrhages and myelin loss were centred in the pontine tegmentum, basis pontis fibre tracts and nuclei, middle cerebellar peduncles, more so on the left side, and in the white matter of the cerebellar vermis. The tegmentum of the medulla and midbrain were midly involved. Mild focal acute inflammatory exudates were present in the meninges of the brainstem. Only one area outside the posterior fossa was involved. It was a microscopic focus showing fibrinoid necrosis of small veins with perivascular acute inflammation. It extended from the tegmentum into the thalamus. The large haemorrhages in the left midbrain tegmentum and head of the right caudate nucleus were interpreted as secondary to brainstem compression and increased intracranial pressure. Severe anoxic changes with early infarcts were found in the left hippocampus. Anoxic neurons were also found in the inferior olivary, XIIth and cuneate nuclei of the medulla. The cerebellar cortex was poorly preserved, with diffuse anoxic Purkinje 


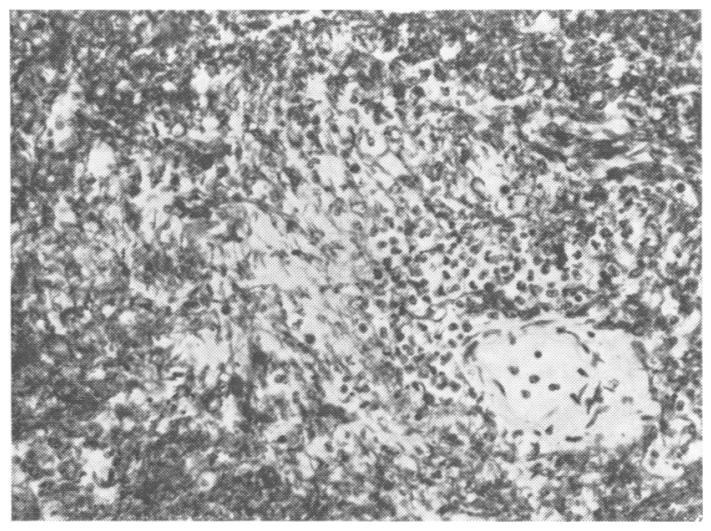

Fig 6 Case 2. Photomicrograph of the left middle cerebellar peduncle showing acute perivascular inflammation and demyelination. Loyez $\times 300$.

cells and conglutination of the granular layer. Fragments of degenerated cerebellar tissue were found in the spinal subarachnoid space.

\section{Discussion}

Morphologically, the diagnosis of acute haemorrhagic leucoencephalitis is based on five main types of lesions, all present in our cases: (1) fibrinoid necrosis of small veins and arteries, with fibrinous exudates extending into the surrounding neural parenchyma, (2) polymorphonuclear leucocytic infiltrates replaced in the later stages by mononuclear cells, (3) multiple ball or ring haemorrhages, (4) perivascular tissue destruction including demyelination and (5) perivascular or confluent oedema. ${ }^{3}$ These changes are often distributed unevenly in the central nervous system. In his original description, Hurst $^{1}$ observed that in each of his two cases the left hemisphere was more involved than the right. This asymmetry, although not always present, has been frequently noted in subsequent reports. Hurst ${ }^{1}$ also reported extension of the lesions into the brainstem and cerebellum. However, lesions exclusively confined to these two structures have been recorded only rarely. The table summarises two previously reported well-documented cases ${ }^{9}$ and the two cases described in this paper. Three of these cases had mild supratentorial involvement probably in continuity with the midbrain lesions. Apart from the less specific symptoms of headache, fever and altered sensorium, cranial nerve dysfunctions and pyramidal signs dominated the clinical picture. The other data fit the general description of acute haemorrhagic leucoencephalitis, with the possible exception of the age of our first patient, who is the first reported older than 60 years. ${ }^{10}$

Two cases, ${ }^{11} 12$ first mentioned by Adams et al ${ }^{9}$ in their review of the literature and regarded by them as consistent with acute haemorrhagic leucoencephalitis, are, after review, excluded. Kaiser's case $^{11}$ was that of a 20 -year-old male who developed an acute vertigo followed three weeks later by ophthalmoplegia, quadriplegia, coma, and death five days later. Predominantly grey matter involvement of the brainstem associated with degeneration of anterior horn neurons in the spinal cord was described, and only microscopic haemorrhages were present. In our view, Kaiser's original diagnosis of an acute ("inferior") polioencephalitis should be retained. The case of Adolf and Spiegel (Case 12, $1922)^{12}$ was in a 43 -year-old female with a gastric carcinoma (metastatic to one ovary at autopsy), who developed headaches, pain in the feet, fever, coma and death fourteen days later. Two foci of non-confluent petechial haemorrhages were present in the white matter of the cerebrum in addition to the brainstem involvement. The microscopic picture consisted of hyaline thickening of the capillary blood vessels and hypertrophic endothelial cells. No

Table Cases of acute haemorrhagic leucoencephalitis involving mainly the posterior fossa structures

\begin{tabular}{|c|c|c|c|c|c|c|c|}
\hline Cases & Sex & Age $(y r)$ & Prodomal illness & $\begin{array}{l}\text { Length of } \\
\text { disease }\end{array}$ & Major symptoms & Structures involved & Comments \\
\hline \multicolumn{8}{|l|}{ Adams et $a l^{9}$} \\
\hline (case 2, 1949) & $\mathbf{M}$ & 34 & $\begin{array}{l}\text { Common cold and } \\
\text { fever, } 1 \text { week }\end{array}$ & 44 hours & $\begin{array}{l}\text { Flaccid quadriplegia, } \\
\text { fever, meningism, } \\
\text { ophthalmoplegia, } \\
\text { cranial nerve palsies, } \\
\text { coma }\end{array}$ & $\begin{array}{l}\text { Midbrain, pons, } \\
\text { superior and } \\
\text { middle cerebellar } \\
\text { peduncles }\end{array}$ & $\begin{array}{l}\text { Subthalamus } \\
\text { involved }\end{array}$ \\
\hline (case 3. 1949) & $\mathbf{F}$ & 56 & None & 30 hours & $\begin{array}{l}\text { Coma at time of } \\
\text { admission }\end{array}$ & Midbrain, pons & $\begin{array}{l}\text { Subthalamus } \\
\text { involved }\end{array}$ \\
\hline Present case 1 (1981) & $\mathbf{F}$ & 82 & None & 6 days & $\begin{array}{l}\text { Headache, seizures, } \\
\text { fever, coma }\end{array}$ & $\begin{array}{l}\text { Brainstem, } \\
\text { cerebellum }\end{array}$ & $\begin{array}{l}\text { Surgery } 2 \text { days } \\
\text { before disease, } \\
\text { biphasic course }\end{array}$ \\
\hline Present case $2(1981)$ & $\mathbf{M}$ & 39 & $\begin{array}{l}\text { Flu-like symptoms, } \\
\text { I week }\end{array}$ & 18 hours & $\begin{array}{l}\text { Fever, dysarthria, } \\
\text { pyramidal signs, } \\
\text { respiratory arrest }\end{array}$ & $\begin{array}{l}\text { Brainstem, } \\
\text { cerebellum }\end{array}$ & $\begin{array}{l}\text { Thalamus } \\
\text { involved }\end{array}$ \\
\hline
\end{tabular}


inflammatory exudate is mentioned. Haemorrhagic skin lesions were also present. The pathological lesions are, in our view, strongly suggestive of a vasculo-haemorrhagic diathesis associated with gastric carcinoma, possibly thrombotic microangiopathy.

Acute haemorrhagic leucoencephalitis may sometimes have a fluctuant or chronic course, especially if cerebral decompression has been performed. ${ }^{4} 6$ In our first case, the evolution was biphasic, with an acute onset in the second day after operation followed by improvement in the following three days. The final hours were, however, fulminant, with death occurring six days after the onset of the symptoms. This biphasic course correlated with the morphologic findings. The foci in the cerebellar white matter with mononuclear infiltrates and few haemorrhages presumably corresponded to the part of the brain affected initially, whereas the brainstem regions showing polymorphonuclear infiltrates and many haemorrhages were involved in the final phase.

The first case is also unusual because the onset of acute haemorrhagic leucoencephalitis followed the implantation of a ventriculoatrial shunt. To our knowledge, this is the second reported case of acute haemorrhagic leucoencephalitis immediately following a surgical procedure. Gosztonyi ${ }^{13}$ reported the case of a 56-year-old male who developed symptoms of the disease two days after repair of incisional and right inguinal hernias, and who died two days later. In these two cases, a causal relationship to operation is difficult to prove and could be coincidental. These patients might have had an undocumented upper respiratory tract infection before surgery. Acute haemorrhagic leucoencephalitis may also occur de novo. In our patient, it is interesting to note that pulmonary infiltrates, shown by chest radiographs, were present during the two acute phases of the disease, but were not in the interval while she was improving. At autopsy, acute bronchitis and patchy bronchopneumonia were found without evidence of a viral or atypical pneumonitis. The latter has been associated once with acute haemorrhagic leucoencephalitis. ${ }^{14}$

A toxic reaction to gentamicin was considered in our first case. It is a routine neurosurgical procedure to inject 4 or $6 \mathrm{mg}$ of this antibiotic through the catheter at the time of surgery. Of the seven cases of gentamicin encephalopathy reported in the literature, ${ }^{15-18}$ all received the drug parenterally, and none were given it intrathecally or intraventricularly. All patients received more than one dose, the total doses ranging from $100 \mathrm{mg}$ to $1470 \mathrm{mg}$. The symptoms seemed to be a combination of seizures, mental changes, and psychologic abnormalities. There were no reported mortalities; all cases improved, usually after discontinuation of the antibiotic.

Watanabe et al ${ }^{18}$ reported neuropathologic changes presumably due to intrathecal gentamicin in a 59-year-old male with multiple sclerosis who received gentamicin $(75 \mathrm{mg}$ intravenously every 8 hours and $5 \mathrm{mg}$ intrathecally every 48 hours over 20 days) for Pseudomonas aeruginosa meningitis. Although there was significant neurological improvement with therapy, he died of congestive heart failure secondary to endocarditis. In addition to the lesions of multiple sclerosis, there were discrete areas of tissue rarefaction disseminated in the brainstem and independent of the sclerotic plaques. In the centre of these lesions, there was a loss of axis cylinders, oligodendrocytes and astrocytes. Along the periphery, there was marked swelling and calcifications of axons. Throughout there was a poor inflammatory response. All the lesions were restricted to myelinated fibre tracts. Similar neuropathological changes were found in the brainstem and cervical spinal cord of rabbits given intracisternal injections of gentamicin at various concentrations. ${ }^{1819}$ These changes presumably were due only to gentamicin, as injections of the carrier substance or preservative did not reproduce them. However, it took doses 50 to 100 times greater than those usually given therapeutically to cause the lesions. These observations conclusively exclude gentamicin as the cause of the morphological changes in the posterior fossa structures of Case 1.

The aetiology and pathogenesis of acute haemorrhagic leucoencephalitis are obscure. The diagnosis is rarely made before death and thorough clinical investigations are rare. There is general agreement that it represents an allergic phenomenon. Delayed hypersensitivity mechanisms were suggested following the demonstration of lymphoblastic transformation in response to encephalitogenic myelin protein. ${ }^{6}$ Also, a hyperacute form of experimental allergic encephalitis produced by pertussis vaccine and adjuvants has been suggested as an experimental counterpart of acute haemorrhagic leucoencephalitis. ${ }^{20}$ The terms disseminated vasculomyelinopathy has been proposed to emphasise the vascular component of the post-infectious encephalitides. ${ }^{21}$ More recently, Reik ${ }^{22}$ expanded this concept with the hypothesis that circulating immune complexes could be the initial event in the vasculomyelinopathies. Our knowledge of the pathogenetic mechanisms in acute haemorrhagic leucoencephalitis is, however, insufficient to explain variability in the localisation of the tissue reaction.

We are grateful to Dr Lucien $\mathbf{J}$ Rubinstein for his useful advice in the preparation of this report 
and his help with the German literature. We thank Mary Ann Lawrence and Robert McGowan for technical assistance, Phil Horne for the photography and Marion Cullen for secretarial help.

One of us (JM) was a grantee of the RS McLaughlin Fellowship Foundation (selection by Laval University Medical School).

\section{References}

${ }^{1}$ Hurst EW. Acute haemorrhagic leucoencephalitis: a previously undefined entity. Med J Aust 1941; 2:1-6.

2 Carpenter S, Lampert PW. Postinfectious perivenous encephalitis and acute hemorrhagic leukoencephalitis. In: Minckler J, ed. Pathology of the Nervous System, Vol. 3. New York: McGraw-Hill, 1972; 2260-9.

${ }^{3}$ Oppenheimer DR. Demyelinating diseases. In: Blackwood W, Corsellis JAN, eds. Greenfield's Neuropathology, 3rd ed. London: Edward Arnold Publication, 1976;490-1.

${ }^{4}$ Coxe, WS, Luse SA. Acute hemorrhagic leukoencephalitis: a clinical and electron microscopic report of two patients treated with surgical decompression. $J$ Neurosurg $1963 ; 20$ :584-96.

${ }^{5}$ Litel G, Ehni G. Acute hemorrhagic leucoencephalitis. Treatment with corticosteroids and dehydrating agents. $J$ Neurosurg 1970;33:445-52.

${ }^{6}$ Lamarche JB, Behan PO, Segarra JM, Feldman RG. Recurrent acute necrotizing hemorrhagic encephalopathy. Acta Neuropathol (Berl) 1972;22:79-87.

${ }^{7}$ Lapham LW, Johnstone MA, Brundjar KH. A new paraffin method for the combined staining of myelin and glial fibers. J Neuropathol Exp Neurol 1964; $23: 156-60$.

${ }^{8}$ Horten BC, Rubinstein LJ. Primary cerebral neuroblastoma. A clinicopathological study of 35 cases.
Brain 1976;99:735-56.

${ }^{9}$ Adams RD, Cammermeyer J, Denny-Brown D. Acute necrotizing hemorrhagic encephalopathy. J Neuropathol Exp Neurol 1949;8:1-29.

${ }^{10}$ Behan PO, Moore MJ, Lamarche JB. Acute necrotizing hemorrhagic encephalopathy. Postgrad Med 1973; 54:154-60.

${ }^{11}$ Kaiser O. Zur kenntniss der polioencephalomyelitis acuta. Dtsch $Z$ Nerven 1895;7:359-91.

${ }^{12}$ Adolf M, Spiegel E. Zur pathologie der epidemischen encephalitis. Arb Neurol Inst (Wien) 1922;23:36-66.

${ }^{13}$ Gosztonyi G. Acute hemorrhagic leucoencephalitis. Report of three cases. Z Neurol 1973;204:43-66.

${ }^{14}$ Fowler M, French EL. A case of acute haemorrhagic leucoencephalitis (Hurst) following atypical pneumonia, with a description of cultural and virus studies. Med J Aust 1949;36 I :267-71.

${ }^{15}$ Byrd GJ. Acute organic brain syndrome associated with gentamicin therapy. $J A M A$ 1977;238:53-4.

${ }^{16}$ Kane FJ, Byrd G. Acute toxic psychosis associated with gentamicin therapy; case report. South Med J $1975 ; 68: 1283-5$.

17 Wadlington WB, Hatcher $\mathrm{H}$, Turner DJ. Osteomyelitis of the patella: gentamicin therapy associated with encephalopathy. Clin Pediatr (Phila) 1971;10: 577-80.

${ }^{18}$ Watanabe I, Hodges GR, Dworzack DL, Kepes JJ, Duensing GF. Neurotoxicity of intrathecal gentamicin: a case report and experimental study. Ann Neurol 1978;4:564-72.

19 Watanabe I, Hodges GR, Dworzack DL. Chemical injury of the spinal cord of rabbits after intracisternal injection of gentamicin. $J$ Neuropathol Exp Neurol 1979;38:104-13.

${ }^{20}$ Levine S, Wenk EJ. Allergic encephalomyelitis: a hyperacute form. Science 1964;146:1681-2.

${ }^{21}$ Poser CM. Disseminated vasculomyelinopathy. Acta Neurol Scand 1969;45: Supplement 37:1-44.

${ }^{22}$ Reik L. Disseminated vasculomyelinopathy: an immune complex disease. Ann Neurol 1980;7:291-6. 